ARTÍCULOS 



\title{
CENTRALIDAD FUNCIONAL Y RECONFIGURACIÓN METROPOLITANA ${ }^{1}$
}

\author{
Amalia Vahí Serrano \\ Virginia Rodríguez Díaz \\ Claudia Hurtado Rodríguez \\ Departamento de Geografía, Historia y Filosofía. Universidad Pablo de Olavide. Sevilla \\ avahser@upo.es
}

\section{RESUMEN}

Nuevos grandes centros de actividad funcional inducen de un modo $\mathrm{u}$ otro a procesos expansivos urbanos que a menudo -lejos de señalarlos como vertebradores de la ciudadresultan proyectos poco o mal integrados. Así, la ciudad metropolitana acusa importantes cuestiones mejorables en la armonización de las piezas, y siempre susceptibles de seguimiento y reorientación. Este artículo propone brindar resultados obtenidos de la identificación de grandes centros de actividad y el papel desempeñado en las transformaciones urbanas metropolitanas. Como fin último se persigue contribuir a la construcción de modelos urbanos sobre la base de un desarrollo equilibrado, en coherencia con la sostenibilidad ambiental.

Palabras clave: ciudades, sistemas metropolitanos, grandes centros funcionales de acti$\operatorname{vidad}$ (G.C.F.A.).

\section{ABSTRACT}

New biggest functional centers of activity in metropolitan areas induce increasingly expansive urban processes and deployment of equipment that often demand new infrastructure elements far from integrated projects, what add often new difficulties to the Metropolis. Some of these requirements are capable of tracking, improvement or reorientation. This paper aims to provide results of the identification of major centres of activity and the role

Fecha de recepción: febrero 2013.

Fecha de aceptación: diciembre 2013.

1 Este artículo se inserta en el Proyecto del Plan Nacional I+D, «Áreas metropolitanas: dinamicas espaciales, sostenibilidad y gobernanza», dentro de Proyecto Coordinado «Movilidad y reconfiguración urbana y metropolitana», cód. CSO2011-29943-C03-01, del Ministerio de Economía y Competitividad. 
played in the metropolitan urban transformations. As ultimate goal aims to contribute to the construction of urban models into a balanced environmental and territorial development.

Keywords: cities, metropolitan systems, major centres of activity.

\section{INTRODUCCIÓN}

La concentración poblacional sobre los espacios urbanos en el último tercio del siglo XX y la primera década del siglo XXI es una constante en muchos rincones del planeta que denota una tendencia variable al crecimiento. Se trata de un crecimiento expansivo de carácter generalizado que ha venido de la mano de múltiples factores sociales, económicos y ambientales, vinculados al devenir histórico. En ese contexto, el crecimiento urbano, con su demanda progresiva de suelo y energía, ha desembocado en el reconocimiento de una escala superior, metropolitana, con nuevos escenarios y actores en los que la presencia de los servicios se ha impuesto espacialmente al resto de las actividades económicas. La nueva ciudad metropolitana del siglo XXI experimenta una conformación y distribución funcional según la cual se reconocen nuevos nodos y áreas de centralidad en torno a grandes equipamientos que atraen no sólo capital e inversiones sino también -en el día a día- algunos de los mayores flujos de desplazamientos sobre la ciudad. El presente artículo plantea la trascendencia de abordar el fenómeno metropolitano, incorporando la complejidad de escalas y el papel de los elementos que interactúan, dirigiendo los autores la mirada y el análisis hacia los grandes centros de actividad que inciden en la nueva ordenación urbana. La incorporación de distintas escalas, sectores de actividad y jerarquías, hace que el tratamiento de los datos y los análisis posteriores requieran planteamientos atrevidos pero firmes y objetivos, capaces de comportar soluciones para nuevos conflictos sobre los nodos, las redes y la movilidad en general.

Las áreas y aglomeraciones urbanas presentan procesos y transformaciones de porte diferente aunque se detectan pautas constantes en numerosos casos, como el hecho de que ciertos servicios (por ejemplo, comercio o atención sanitaria a determinados niveles), recurren a emplazamientos periféricos en el plano de la ciudad central asociados directa e indirectamente a la malla del viario que soporta la movilidad de alcance metropolitano. Descifrar sus pautas y relaciones ayuda a entender determinados patrones de crecimiento y funcionamiento en las áreas metropolitanas, lo que a su vez puede comportar aprobación o revisión de la gestión urbana emprendida, a partir del seguimiento y evaluación. En definitiva, se trata de minimizar las tensiones planteadas en estos espacios, de ahí el interés del presente artículo.

La necesidad de dar respuesta a la presión demográfica y las actividades económicas asociadas a la misma han devenido en la consolidación de unos espacios fuertemente ocupados que ponen en entredicho la adecuación de los suelos u otros recursos al modelo de demanda actual. Una mirada a esta parte del planeta y a las estadísticas conocidas permite conocer que Europa cuenta con 47 grandes aglomeraciones con -al menos- un millón de habitantes (de las 498 que existen en el mundo a comienzos de 2013), y muchas de ellas alcanzaron esa cifra desde 1950 en adelante. El modelo demográfico reproducido desde entonces es el reflejo de una región libre de inestabilidades bélicas (excepción hecha de la situación balcánica) y -a pesar de la crisis económica financiera desatada en 2008- con una potente política de crecimiento económico diseñado por la UE a instancias de los países miembros. 
En España, más de doce millones de habitantes se distribuyen sólo entre cuatro aglomeraciones españolas (Madrid, Barcelona, Valencia y Sevilla) según fuentes de diferente índole ${ }^{2}$, aunque nuestra intención recoge un espectro más amplio. La tendencia histórica, con frecuencia, y las coyunturas recientes (no siempre previsibles) en diferentes escenarios, han dado lugar a procesos de densificación poblacional y económica que demandan toda la atención por parte de quienes administran la ciudad y el territorio. Dichos procesos están claramente identificados dado el interés en la búsqueda de soluciones a algunos de los principales problemas sobrevenidos (multiplicación de los desplazamientos cotidianos y consiguiente motorización excesiva, pérdida de calidad ambiental, inadecuación de los suelos a los usos a los que se están destinando, etc.). Con carácter general, Europa también se hace eco de la evolución seguida, como muchos de sus informes y publicaciones manifiestan (véanse los Libros Blancos de transportes, el Libro Verde sobre la movilidad urbana,...). Realmente el interés y la investigación sobre estas cuestiones tienen un recorrido no muy largo y surgen casi en simultáneo a procesos precipitados por la evolución de la población, las actividades económicas y la ocupación de los suelos.

Si bien es cierto que no existe en nuestro país una delimitación oficial, en el tiempo se han aplicado criterios por parte de las instituciones públicas (Atlas Estadístico de las Áreas urbanas en España, Ministerio de Vivienda; 2007; publicaciones del Observatorio de la Movilidad Metropolitana, Ministerios de Fomento y Medio Ambiente), basados en el tamaño y algunos criterios espaciales que no terminaban de satisfacer la necesidad de cohesión demandada por los propios ámbitos afectados. A lo largo de la década del 2000, se han realizado avances sobre los criterios de delimitación de las áreas metropolitanas y la metodología de trabajo, en consonancia con los que se emplean y utilizan en otros países del mundo desarrollado (Feria, 2004; Casado, Coombes, 2005).

La necesidad de resolver esta cuestión no es algo baladí, ya que de modo cada vez más insistente y acuciante, se detecta una demanda por parte de las administraciones públicas para disponer de áreas relevantes para afrontar la dimensión inferior a las regiones y las provincias. Los resultados de investigaciones recientes han permitido trazar con precisión el mapa de las áreas metropolitanas en España, de acuerdo con una metodología compleja contrastada y reconocida (Borruso, 2003; Feria, 2008; Salom, Albertos, 2010). De acuerdo a los criterios contrastados y en consonancia con las fuentes disponibles, en España se registran 46 áreas metropolitanas (Figura 1) con una población censada de 31.745.459 habitantes ${ }^{3}$. Esta cifra encierra dos hechos significativos: representa el 67,27 \% de la población española, y la superficie total de las áreas alcanza el $14 \%$ de suelos del territorio del Estado. España es el cuarto país más poblado de Europa (por detrás de Alemania, Francia y Reino Unido), aunque su densidad poblacional se encuentra por detrás de todos los países centroeuropeos y otros de la región meridional como Italia o Portugal.

2 En nuestro proyecto trabajamos con datos censales sobre los cuales establecemos la agregación e identificación de las áreas metropolitanas, que acaban resultando mayores que las recogidas en otras fuentes no oficiales pero ilustrativas, como http://www.citypopulation.de. - Principal Agglomerations of the World producto de libre acceso en internet, creado por Th. Brinkhoff. Se nutre de estadísticas oficiales institucionales muy diversas sobre cuya lectura es preciso identificar heterogeneidad y no equivalencia de las variables.

3 Censo 2011. Cifra que ha de tomarse con la precisa cautela en este momento en que todavía el Instituto Nacional de Estadística no ha publicado los resultados definitivos. 


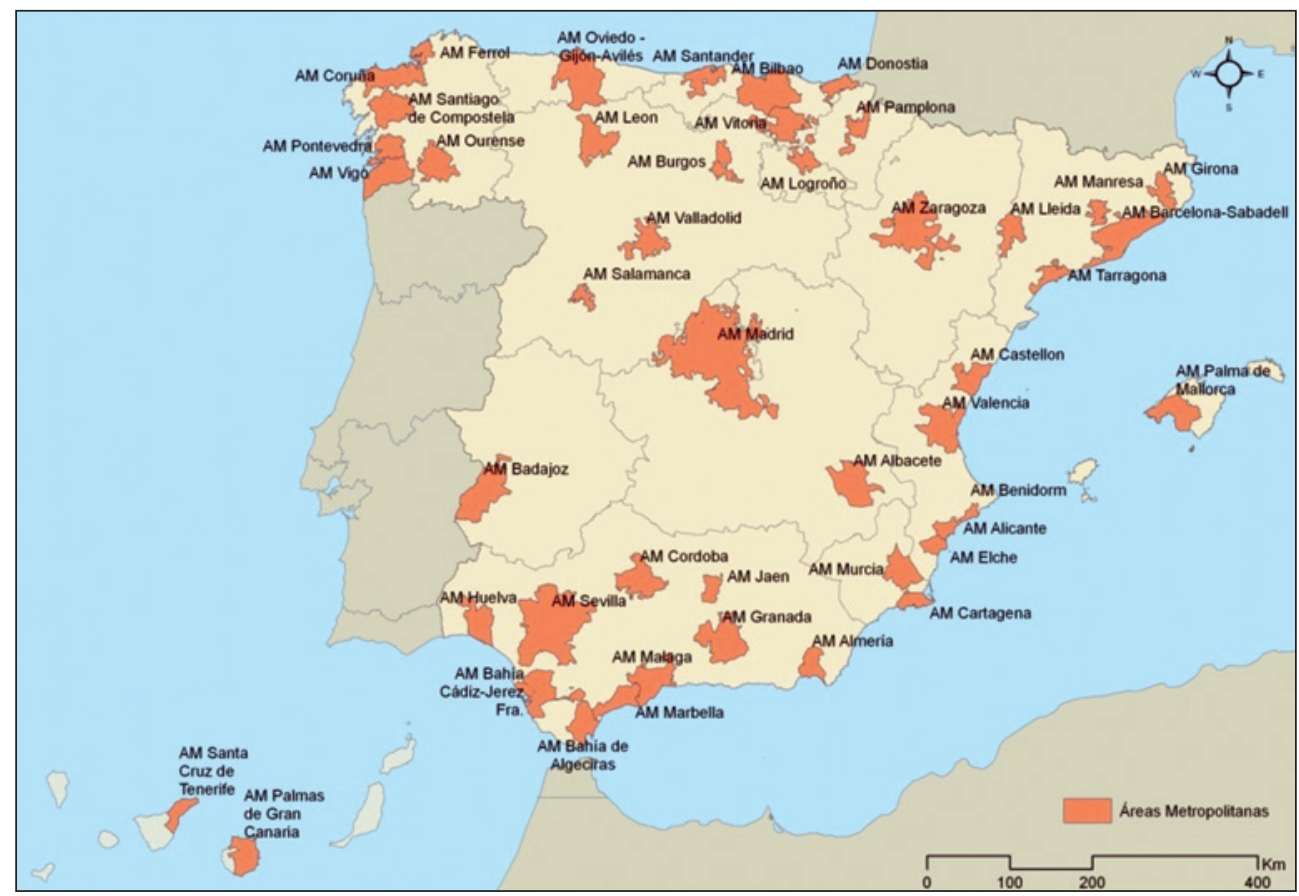

Fuente: elaboración propia a partir de Feria, J.M. (2008).

En los umbrales de la segunda década del siglo XXI se identifica una gran diversidad de tamaño (población y extensión) y dinámica (mercados, movilidad cotidiana, usos de suelo,...) de las áreas españolas. Dicha diversidad y complejidad engrosan el conocimiento e información del fenómeno metropolitano en nuestro país, y en consecuencia hoy se conocen resultados relevantes sobre la génesis, variaciones y repercusiones desde perspectivas interdisciplinares (Feria, Albertos, 2010) sobre las que es preciso profundizar. En consonancia con ello, la caracterización metropolitana, (demográfica, aunque no en exclusiva) contribuye a las propuestas de ordenación territorial y urbanística a la que no escapa la planificación de los servicios (Vinuesa, 2012). Sobre esta base de diversidad y complejidad de la realidad analizada, el artículo trata de identificar el papel y alcance que desempeñan grandes equipamientos en la configuración metropolitana en nuestro país.

\section{LOS GRANDES CENTROS FUNCIONALES DE ACTIVIDAD, VERTEBRADORES URBANOS}

La delimitación de los grandes centros funcionales de actividad como vertebradores urbanos se inserta en una concepción integral de la realidad metropolitana compleja, al detectarse que estos elementos se incorporan a menudo al planeamiento obviando o infravalorando sus capacidades para configurar nuevos espacios urbanos o reformular los existentes. 
Los espacios urbanizados en España resultan de sucesivas fases de expansión que en la segunda mitad del siglo XX se intensificaron. Con carácter diferenciado y en múltiples direcciones según los casos, el mercado inmobiliario impulsó un crecimiento centrífugo sin precedentes que ha acabado por unir piezas, sectores y núcleos de población (antaño diferenciados), y todo ello mediante procesos de suburbanización y periurbanización que han dado lugar a la metropolitanización. El rol que juegan determinadas actividades a través de su localización y dimensión no ha pasado desapercibido en tiempos recientes, cuando los efectos de esa dinámica metropolitana se han hecho sentir. En ese sentido son referencias ineludibles los análisis que para los casos de Madrid o Barcelona han realizado algunos investigadores, fundamentalmente centrados en las actividades industriales, comerciales y de distribución (Méndez, Gutiérrez Puebla, entre otros) ${ }^{4}$. La premisa fundamental de este texto sitúa a los G.C.F.A. como inductores, en parte, de la movilidad metropolitana y por tanto como interlocutores dentro del planeamiento en la resolución de tensiones y respuestas, al menos del mercado inmobiliario, las infraestructuras de las comunicaciones y los servicios de transportes.

En la escala supralocal los patrones de movilidad frecuente de la población conducen a rediseñar los sistemas generales y determinados servicios con la consiguiente garantía de -al menos- proximidad y accesibilidad. La oportunidad brindada por dichos cambios, al servicio del crecimiento urbano, sirvió para reorganizar la dotación de determinados equipamientos, contribuyendo al mismo tiempo a redimensionar la oferta comercial, con tamaños de establecimientos superiores a los tradicionales y una organización espacial conectada a las nuevas necesidades que iban surgiendo (Vahí, 2007). Paulatinamente, la sanidad y la educación fueron siendo subsumidas por el modelo sociopolítico que abría los servicios a una mayor cantidad de población con lo que la titularidad privada entró en competencia con la pública, y de ahí se pasó a la construcción de hospitales y universidades que demandaban grandes solares en consonancia con la oferta que se proponía garantizar. Simultáneamente, en ocasiones de modo tardío, los polígonos industriales preexistentes fueron dando paso a espacios productivos de nueva generación y criterios urbanísticos (diversidad tipológica de polígonos y parques empresariales,...), con la consiguiente aparición de los centros logísticos y centros intermodales de transportes. Desde los años 80 en adelante, la nueva versión de espacios productivos se prodigó por la geografía española hasta el punto de surgir nuevos polígonos y parques rompiendo con frecuencia la racionalidad urbanística y ambiental. De la mano de las propias empresas (o en ocasiones inducidas por agentes externos como la universidad, la administración pública,...) se produjeron nuevos progresos al incorporar las claves de la innovación e investigación para la mejora de la calidad en procesos y productos, algo que indujo a la dotación de espacios de centralidad donde convergieran entidades implicadas en I+D+I e interesadas en compartir sinergias, recursos y oportunidades sobre un mismo espacio con la consiguiente reducción de costes. Muy sintéticamente, puede decirse que así nacieron buena parte de los parques tecnológicos en España.

4 De modo específico el trabajo de Gutiérrez, J. y García, J.C. sobre descentralización de actividades económicas en Madrid es una de las referencias básicas en nuestro trabajo (vid. Bibliografía). 
La identificación de estos nodos, espacios de centralidad activa, en periferias y territorios metropolitanos ${ }^{5}$ conlleva la tarea de desagregar usos y elementos integrados en ellos así como descifrar su papel sobre el sistema funcional urbano municipal o supralocal y, sobre todo, interpretar el alcance de las fortalezas y debilidades que se desprenden de tan compleja dinámica. En la perspectiva científica, el objetivo debe ser determinar la minimización de amenazas o marcar cauces de las oportunidades que se desprendan de los G.C.F:A. como claves en la reconfiguración metropolitana desde la complejidad de la evaluación ambiental estratégica. Dado el mayor recorrido que tienen las investigaciones en torno a los espacios productivos en nuestro país, y la relativa menor incidencia de estos sobre la movilidad cotidiana, centraremos la atención en los grandes centros comerciales y otros servicios como los equipamientos públicos de nivel superior (universitarios y hospitalarios) y otros centros de distribución (logísticos y de transportes).

Estos equipamientos funcionales han sido abordados desde diferentes perspectivas, especialmente desde finales de los años 90, conforme se fueron asentando y madurando. Referencias relevantes sobre grandes tipologías comerciales y espacios urbanos se encuentran en Pozo, Ruiz, Lázaro (2000); Tornos, coord. (2000); Villarejo, Álvarez (2004), Fernández, García (et al.) (2003), Escudero (2008), Vahí, Feria (2007). Los restantes servicios identificados, menos explorados en su componente territorial y relacional, han alimentado no obstante algunas vías de trabajo y no faltan referencias, como Rodríguez (2010), Caravaca, Feria (2005) entre otros. La presente propuesta se incardina en el debate sobre los escenarios en los que la centralidad funcional acotada más arriba incide en la vida cotidiana a causa del complejo ensamblaje entre emplazamientos, desplazamientos, infraestructuras y medios de transportes.

\section{METODOLOGÍA}

El alcance de los G.C.F.A., como vertebradores urbanos e inductores de la movilidad metropolitana, cubre el propósito del presente texto y se convierte en uno de los objetivos del amplio proyecto mencionado con anterioridad. El denominador común de estos grandes centros de actividad es, junto a la dimensión, el carácter nodal que se proyecta en el plano sobre la ciudad nuclear y el conjunto metropolitano, con alcance incluso (gradualmente debilitado) más allá de éste. El hecho diferencial no sólo radica en la orientación y sustantividad del servicio que ofrecen (salud, educación, investigación, apoyo logístico al transporte) sino también en la caracterización que gozan dentro de una jerarquía y un modelo de gestión como veremos.

La información necesaria inspira el diseño de una base de datos cuya estructura recoge caracterización de tipologías, identificación, tamaños, localización y otros rasgos sustanciales para nuestro propósito. La geocodificación consiguiente se acompaña de labores de contraste y confirmación de determinados registros, y una primera aproximación a la adecuación de los emplazamientos señala a una desigual relación de los mismos con la lógica urbanística de escala supralocal.

5 Para comprender el sentido dado a la periferia urbana y el territorio metropolitano, véase Vahí, A. (2007), vid. en Bibliografía. 
La cartografía permite constatar el papel y alcance de estos centros no sólo en la dinámica urbana expansiva (en términos absolutos y por grandes sectores urbanos) sino también en la interpretación del propio modelo de territorio que se propone desde las administraciones implicadas. La localización en ocasiones descentrada o fuera del entramado y la red metropolitana suscita una mirada retrospectiva. No es casualidad el registro de algunos Parques Tecnológicos y Científicos en emplazamientos más remotos de las coronas metropolitanas, frente a las preferencias de los grandes equipamientos del comercio o la sanidad, que requieren priorizar unas garantías básicas de comunicaciones para flujos más abundantes y regulares de usuarios. Realmente frente a estos, aquellos Parques no están arriesgando sino que podría inferirse todo lo contrario, con su localización más distante de la centralidad urbana. De éstas y otras cuestiones se hace eco la presente investigación, pero veamos en detalle algunos aspectos metodológicos que conviene matizar y caracterizar en función de la tipología y el servicio que brindan estos centros funcionales.

\section{III.1. Instrumentos y fases de trabajo}

Como ya hemos introducido, el papel vertebrador de los G.C.F.A., unido a la tendencia cada vez mayor hacia la localización de estos centros en la periferia urbana, hace que el trabajo de localización exacta de estos elementos en el territorio sea de suma importancia.

En esta línea, la aportación de este trabajo no radica tanto en el uso de una serie de herramientas novedosas, como el valor añadido que brinda la posibilidad de llevar a la práctica un modelo metodológico que nos permita explorar la relación espacial, pero también temporal, de estos centros de actividad con la trama urbana, y que nos ayude a entender un poco mejor de qué modo estos grandes centros polarizadores contribuyen a la construcción del territorio metropolitano.

Teniendo en cuenta esta serie de cuestiones, el ensayo metodológico de este trabajo está diseñado de modo que se pueden identificar cuatros fases: (i) delimitación de las áreas metropolitanas como ámbito de estudio (trabajo ya realizado), (ii) identificación de los G.C.F.A. según las fuentes de información (directorios de origen) y aplicación de criterios de selección, (iii) diseño e introducción de centros (nodos) en base de datos geográfica y (iv) explotación de base de datos (análisis espacio-temporal).

Dentro de este proceso, que se sintetiza en la figura 2, cabe destacar la necesidad de un trabajo exhaustivo de consulta de los centros en los distintos directorios y de verificación de la información disponible, así como la importancia, debido al volumen de datos manejados, de automatizar el proceso de geolocalización para poder hacerlo de forma masiva y de diseñar una base de datos geográfica que posibilite trabajar de forma óptima con tal cantidad de información.

Por otro lado, hay que tener en cuenta que, si bien el proceso metodológico es común para todos los elementos, las distintas tipologías de centros que consideramos como G.C.F.A. obligan a definir ciertos rasgos caracterizadores propios y por tanto a definir unos criterios de selección específicos para cada tipo de centro (tabla 1), del mismo modo que las fuentes de información consultadas son diversas. Es por tanto importante especificar, dentro del proceso metodológico común, una serie de aspectos diferenciadores. 
Figura 2

DIAGRAMA DE FLUJO DEL PROCESO METODOLÓGICO

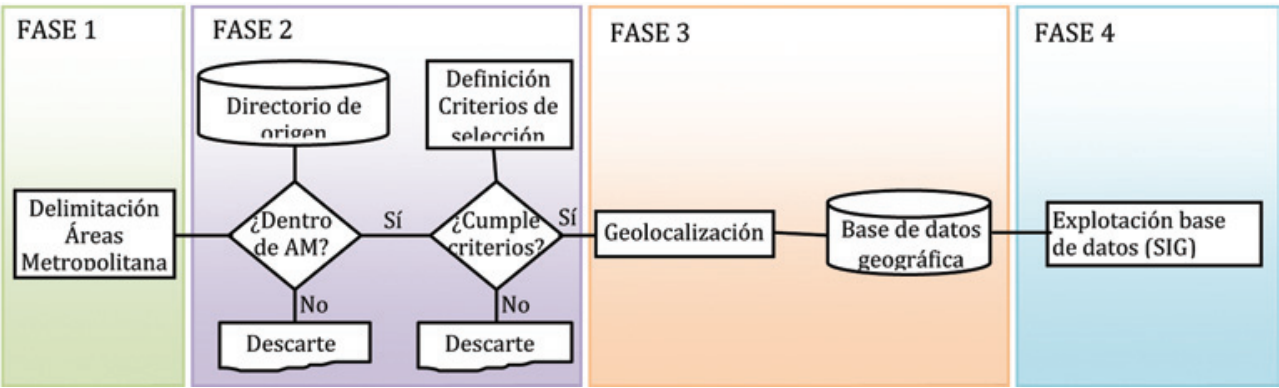

Elaboración propia.

Tabla 1

PROPUESTA DE CRITERIOS ESPECÍFICOS, SEGÚN TIPO DE CENTRO (ACTIVIDAD), PARA TRATAMIENTO DE LA INFORMACIÓN

\begin{tabular}{|l|l|}
\hline \multicolumn{1}{|c|}{ TIPO } & \multicolumn{1}{c|}{ CRITERIO DE SELECCIÓN } \\
\hline CENTROS COMERCIALES & SBA $>5.000 \mathrm{~m}^{2}$ \\
\hline CENTROS HOSPITALARIOS & TITULARIDAD PÚBLICA \\
\hline PARQUES TECNOLÓGICOS & $\begin{array}{l}\text { SOCIOS Y AFILIADOS, ASOC. PARQUES TECNOLÓGI- } \\
\text { COS DE ESPAÑA }\end{array}$ \\
\hline COMPLEJOS UNIVERSITARIOS & $\begin{array}{l}\text { TITULARIDAD PÚBLICA, CONCENTRACIÓN DE OFER- } \\
\text { TA ACADÉMICA (al menOS un grado oficial) }\end{array}$ \\
\hline CENTROS LOGÍSTICOS & COINCIDENCIA EN WEBS INSTITUCIONALES \\
\hline
\end{tabular}

Elaboración propia.

\section{a) Centros comerciales}

A diferencia de los otros tipos analizados, los centros de actividad que gravitan en torno a grandes superficies comerciales se sumen con frecuencia en ciertas ambigüedades que convienen aclarar para la sistematización de los datos. Ello es sumamente útil en nuestro proyecto, más aún si se tiene en cuenta que están sujetos a criterios de localización a menudo superpuestos o con una débil frontera respecto a otros servicios. Ocurre de un modo claro y evidente en los espacios de periferia urbana y territorio metropolitano, donde la dotación de infraestructuras básicas de las comunicaciones permite la convivencia entre la actividad comercial a un determinado nivel y otras actividades como los servicios empresariales y personales (no propiamente comerciales), o incluso establecimientos institucionales públicos. Sobre las coronas metropolitanas que aglutinan estas zonas (periferia urbana, territorio metropolitano) se identifican Parques Comerciales y Parques Empresariales que con frecuencia se hibridan y se complementan. Por otro lado, y así se ha registrado para su posterior análisis, en varios casos se detecta una superposición de aquellos Parques y otras fórmulas de concentración, en lo que ocasionalmente se reconocen parques tecnológicos (a su vez identi- 
ficados puntualmente como partes de diversos campus universitarios). Todas estas cuestiones plantean ocasionalmente dudas razonables acerca de la precisión con la que se denomina a cada elemento de un modo y no de otro, siendo necesario determinar un criterio para el manejo y gestión en nuestro propósito. Para el presente trabajo se ha optado por incorporar los registros conforme al grado de institucionalidad reflejado por las fuentes consultadas.

\section{a) Tratamiento de los datos}

Los establecimientos comerciales están registrados en su mayoría por la AECC en su Directorio periódico (para este trabajo se consultan la edición de 2010) aunque la existencia de muchos centros al margen de la Asociación ha demandado una búsqueda diversificada mediante tareas de campo, consulta en la red y correspondencia con algunas de las enseñas para recabar información respecto a los no socios de AECC. Es ineludible mencionar una de las claves explicativas sobre la demora de los trabajos para la fase de identificación de determinadas grandes superficies. La dificultad que presentan los Grandes Almacenes (de acuerdo a la taxonomía que la propia AECC utiliza) se asienta en la ausencia de datos y/o registros publicados en torno a superficie o tamaño de los establecimientos. Para solventar las primeras barreras que presentan estos casos se ha procedido a su localización en varias fases: a partir de la consulta a la web comercial de la firma sobre el listado de tiendas mediante la ortofoto actualizada (PNOA) ${ }^{6}$ y la correspondiente consignación de datos espaciales. Finalmente se ha discriminado conforme a los criterios comunes para el resto de establecimientos comerciales, excluyendo: aquellos que no alcanzan $5000 \mathrm{~m}^{2}$; aquellos otros que se encuentran insertos en otros grandes centros comerciales (ya registrados); o aquellos que están en los centros urbanos, próximos a una gran tienda de la misma firma, ya reflejada en nuestra base de datos ${ }^{7}$. El resultado es de más de 100 establecimientos que quedan fuera del Directorio AECC y que finalmente son incorporados a nuestra base de información dado el papel que suscitan sobre el ámbito urbano consolidado, responsables en cierta medida (inductores) de las dinámicas de movilidad en los centros de las ciudades en las que se encuentran.

\section{b) Criterios de selección. Geolocalización}

Del análisis de los datos volcados en el Directorio de AECC resultan esclarecedoras algunas conclusiones. Los 500 centros comerciales inicialmente registrados son revisados en coherencia con nuestros criterios de centralidad: en ocasiones, el carácter integrado de algunos de estos centros, caso de algunos Parques Comerciales (bien por estrategia comercial o por autorización/licencia autonómica/urbana), arroja datos de una o más grandes superficies sobre una misma área o parcela urbana. Ese primer filtro los rebaja a 476 centros, que quedarán en 429 al aplicarse el filtro por tamaño (excluidos $10 s<5000 \mathrm{~m}^{2}$ ). La suma de registrados y excluidos del Directorio arroja la cifra de hasta 500 Centros Comerciales (en permanente

6 Ortofoto de máxima actualidad, PNOA (Plan Nacional de Ortofotografía Aérea), consultado 2012.

7 Se trata de un recurso que conduce a ponderar la centralidad que generan estos grandes almacenes allí donde se intensifica su presencia por la suma de varios establecimientos medianos o grandes. La dimensión recogida es la mayor, estando sujeta a una revisión si finalmente se valora reflejar la presencia real de estos establecimientos, localizándolos en el plano y sumando las superficies de todas las tiendas concentradas. 
revisión), de los que aún se pueden añadir alguno(s) más una vez aplicados todos los filtros seleccionados y hecha la revisión de todas las fuentes a nuestro alcance.

Tabla 2

CLASIFICACIÓN DE G.C.F.A. DEDICADOS AL COMERCIO MINORISTA EN LAS ÁREAS METROPOLITANAS ESPAÑOLAS

\begin{tabular}{cll}
\hline $\mathbf{N}^{0}$ CENTROS & TIPOLOGÍA & RANGO SBA $(\mathbf{m} 2)$ \\
\hline 243 & C.C. PEQUEÑOS & $5.000-20.000$ \\
109 & C.C. MEDIANOS & $20.000-40.000$ \\
70 & C.C. GRANDES & $40.000-80.000$ \\
24 & C.C. MUY GRANDES & $>80.000$ \\
54 & S/D & Se desconoce la superficie de la parcela \\
\hline
\end{tabular}

Fuente: Directorio AECC 2012. Elaboración propia.

Sobre los datos del Directorio de AECC se establecen comprobaciones básicas sobre la imagen de ortofoto para corroborar la información, al mismo tiempo que se prevé obtener información de aquellos que no están registrados en dicha fuente, mediante la observación directa, contraste de imágenes y consultas a los centros de los que se desconocen aún una o más variables (tamaño, año apertura,...). Junto con los parques empresariales, estos son elementos presentes en todas las áreas metropolitanas en estudio, y constituyen uno de los resortes -si no el que más- a la hora de la dinamización de los suelos productivos y -por contagio- de la totalidad de los suelos urbanos, por encima de otras opciones menos frecuentes. No obstante, habrá que determinar el valor y el peso que tiene la variable «número de establecimientos» frente a la superficie que ocupa cada uno de ellos, una cuestión que se aborda más adelante.

\section{b) Hospitales}

A la hora de volcar y tratar la información referente a los centros hospitalarios se considera relevante exponer las particularidades del rigor que demanda este tipo de centros de actividad ya que se plantean criterios de centralidad funcional bien distintos de los centros comerciales, y que se explican a continuación. Como objeto de estudio se seleccionan de la red de centros asistenciales los hospitales tanto públicos como concertados ${ }^{8}$ (ya que atienden a usuarios de los sistemas sanitarios de las distintas comunidades en los casos en los que se aprecia déficit asistencial por parte de la red pública).

Otra cuestión a tener en cuenta en la identificación de los hospitales a incluir en el análisis es que en ocasiones la asistencia especializada se organiza en Complejos Hospitalarios formados por dos o más hospitales que comparten órganos de dirección o gestión de presupuestos y -en este sentido- para aquellos complejos hospitalarios que presenten una misma localización geográfica se opta por incluir un único centro en el análisis (normalmente el

8 Se entiende por concierto un contrato realizado entre un establecimiento sanitario (público o privado) y el servicio sanitario autonómico por el que se determinan, bajo ciertas condiciones, las características y las tarifas de los servicios sanitarios que afecta. 
Hospital General ${ }^{9}$ ). En los casos en los que los hospitales del complejo presenten diferentes localizaciones geográficas se estudia la posibilidad de incluir los centros dependientes del complejo según su finalidad asistencial y actividad asistencial.

Los datos relativos a la tipología de hospitales (identificación del centro, finalidad asistencial y dependencia funcional y patrimonial y dotación de camas) se obtienen del Catálogo Nacional de Hospitales 2012 (MSSSI, 2012) por constituirse como la base de datos de carácter oficial que recoge la información relativa a los hospitales de los diferentes servicios de salud de las Comunidades Autónomas. En cuanto a los datos de actividad asistencial se obtienen de la base de datos «Estadística de Establecimientos Sanitarios con Régimen de Internado» (MSSSI, 2012) ${ }^{10}$ que se constituye como la única fuente de información de recursos y actividad de asistencia especializada que cubre todo el ámbito nacional.

\section{a) Tratamiento de los datos}

Para determinar la capacidad que los centros hospitalarios tienen de generar movilidad es imprescindible identificar la demanda, que en nuestro caso se identifica con una demanda potencial ya que nos encontramos ante una asistencia sanitaria universal como eje central del Sistema Nacional de Salud. Por tanto la demanda queda definida por la propia ordenación sanitaria vigente que asocia a cada centro hospitalario su población adscrita, es decir el conjunto de municipios que cuentan con un mismo hospital de referencia. Esta cuestión se complica por la propia organización funcional de la asistencia especializada ya que dependerá de la cartera de servicios que presente cada uno de los centros según el siguiente esquema en orden de nivel de especialización: Hospital Regional, Hospital de Especialidades, Hospital Comarcal y otras carteras de servicios (por ejemplo, Hospitales de Alta Resolución en el caso de Andalucía).

En cuanto al dimensionamiento de los centros hospitalarios debemos tener en cuenta que el proceso de transformación de la asistencia especializada producido en España desde los años 90 ha repercutido sobre el concepto tradicional de hospital desde un centro con internamiento al «ámbito en el que se realiza la atención especializada» (OLLERO, 2004). Esta idea se apoya en la revisión de la actividad ambulatoria en los centros hospitalarios y es especialmente importante en un sistema de salud en el que los servicios de atención especializada suponen el 51,28\% del gasto sanitario público corriente (Estadística del gasto sanitario público, Ministerio de Sanidad, Servicios Sociales e Igualdad, 2013). Al gasto público hay que unir la existencia de una elevada proporción de ingresos y estancias hospitalarias inadecuadas y consultas ambulatorias masificadas y con largas listas de espera.

Unido al cambio en la concepción de las consultas externas surgen nuevos modelos de asistencia basados en el concepto de consulta única o «proceso asistencial ambulatorio en el que queda establecido un diagnóstico junto con su correspondiente tratamiento y reflejados ambos en un informe clínico, siendo realizadas estas actividades en una sola jornada y en un tiempo aceptado por el usuario tras recibir la información oportuna» (Zambrana, 2002).

9 Hospitales destinados a la atención de pacientes afectos de diversa patología y que cuentan con las áreas de medicina, cirugía, obstetricia y ginecología y pediatría. También se considera general cuando, aún faltando o estando escasamente desarrollada alguna de estas áreas, no se concentre la mayor parte de su actividad asistencial en una determinada.

10 La consulta es sobre la más reciente actualización de datos en la web, que se hizo en 2009. 
Este concepto de organización y gestión permite la incorporación de alternativas a la hospitalización tradicional (Cirugía Mayor Ambulatoria -CMA-, hospital de día, hospital de alta resolución) que nos llevan a considerar el número de camas como criterio incompleto en el dimensionamiento de los centros hospitalarios.

\section{b) Criterios de selección. Geolocalización}

Por lo que respecta a los centros hospitalarios tras la discriminación aplicada en función de los filtros aplicados, las primeras cifras son:

Tabla 3

CLASIFICACIÓN DE G.C.F.A. DESTINADOS A HOSPITALES EN LAS ÁREAS METROPOLITANAS ESPAÑOLAS

\begin{tabular}{lll}
\hline 600 & TRAS PRIMER FILTRO & COMPLEJOS GRANDES, 1 O MAS ESTABLECIMIENTOS, \\
\hline 375 & TRAS SEGUNDO FILTRO & CENTROS PÚBLICOS O CONCERTADOS \\
\hline & DE ELLOS: 37 & CON «CERO» CAMAS \\
\hline
\end{tabular}

Fuente y elaboración propia.

La definición de otros filtros relativos a la identificación de la demanda de los centros así como a su dotación y actividad asistencial mediante datos de frecuentación permitirá una mayor especificidad sobre la centralidad que ejerce cada uno de los registros contenidos.

\section{c) Complejos universitarios}

Históricamente la universidad gozó en España de una localización bien centrada sobre el plano urbano. Habida cuenta del escaso número de instituciones fundadas siglos atrás, el encontrarse en un entorno simbólico y representativo de las ciudades acabó por constituirse en referente mismo de éstas (Salamanca, Alcalá de Henares, Sevilla,...) ${ }^{11}$. En la actualidad la situación ha cambiado sustancialmente y el criterio locacional sigue pautas seguidas en otros países europeos, y en consonancia con la mayoría de universidades americanas. Hasta mediados de los 50 del pasado siglo, el Estado promovía en nuestro país la investigación y la creación de centros públicos que albergase dicha actividad, algo que generalmente se localizaba en los centros urbanos. Desde los años 70, sin embargo, las universidades y determinadas empresas comenzaron una andadura juntos (más tímida al comienzo, especialmente en el entorno de las ingenierías y ciertas áreas de las ciencias puras) que suscitaron la oportunidad de localizarse en nuevos entornos que les proporcionaran una nueva identidad, más moderna y renovada, con nuevos criterios edificatorios y de equipamientos funcionales. Antes de acabar la década de los 90, un gran número de ciudades españolas contaban con dotaciones universitarias de carácter periurbano o claramente asentadas en suelos de los municipios de las coronas metropolitanas.

Hoy día, como insisten algunos autores, se han multiplicado los productores de conocimiento identificándose, en ocasiones con dificultad, distintos tipos de servicios en universidades, empresas, institutos, centros tecnológicos, etc. (Sanz, Cruz, 2001).

11 Una visión sintética pero completa de la historia de la Universidad española se encuentra en Atlas digital de la España universitaria (Reques, P., Dir.), 2006. 
La información en esta fase arroja un primer balance acerca de la transformación radical que ha protagonizado el ámbito universitario. Al margen de otras consideraciones sociológicas o de otro tipo, la Universidad se identifica hoy sobre el plano urbano como un gran centro de actividad con una oferta formativa creciente pareja a espacios de investigación, al tiempo que ha ido haciéndose más y más compleja, demandando una estructura organizativa, administrativa y de servicios asociados. La accesibilidad y las buenas comunicaciones son determinantes en la implantación de este tipo de equipamientos que, al igual que los restantes aquí analizados, suscitan una serie de demandas espacio temporales singulares. La afluencia regular e intensa tiene unas cotas características (de lunes a viernes/sábado, y de 8 a 22 de la noche) diferentes a hospitales (continuo, día y noche) y centros comerciales (de lunes a sábado/domingos, horarios más flexibles en función de ocio, restauración,...).

\section{a) Tratamiento de los datos}

Inicialmente se aborda la oferta universitaria de titularidad pública aunque no se descarta la valoración y posterior incorporación de las universidades de carácter privado. Un segundo filtro se aplica en función de la primacía del establecimiento o conjunto, quedando registrados los grandes equipamientos universitarios que concentran la oferta académica, o parte de ella, así se diferencian equipamientos universitarios son subsumidos en la centralidad de algunos de los Parques Tecnológicos, donde la actividad primacial es la investigación (y no la docencia). Este proceso ha tenido un ritmo cambiante hasta su conclusión, pasando por las consultas telefónicas, la identificación in situ y el contraste de datos en webs institucionales.

El Atlas digital de la España universitaria constituye un compendio de información sobre la institución a nivel del país y por universidades, en este caso con indicadores genéricos pero también con algunos datos relativos (por ej./100 estudiantes) sobre los que se explorará más adelante (dotación de aparcamientos para vehículos particulares en los campus universitarios y algunas otras variables). La web institucional de rango superior, Ministerio de Educación, no proporciona datos desagregados de carácter espacial, y las consultas dirigidas al Ministerio se solventaron con la web institucional de la red UNIVERSIA. Su fuente de datos (http://estudios.universia.net/espana/institucion) proporciona información a nivel de universidad y campus, junto a otros datos (no trascendentes de momento en nuestro propósito, pero no descartables): $\mathrm{n}^{\circ}$ de Titulaciones Oficiales de Grado, $\mathrm{n}^{\mathrm{o}}$ de Masteres Oficiales, $\mathrm{n}^{\mathrm{o}}$ de suborganismos (Departamentos, Centros, Facultades, Escuelas, Institutos, etc.).

El campus se considera el nivel de desagregación más acertado al permitir visualizar el poder de atracción de estos complejos a escala metropolitana. El campus suscita una centralidad a partir de la agregación de facultades, centros y servicios varios (administrativos, deportivos, institutos,...), y ese rol aglutinante permite reconocer diferentes niveles de atracción, incluso para una misma universidad, justamente en función de su descentralización.

\section{b) Criterios de selección. Geolocalización}

Sobre la información recopilada en las webs institucionales y contrastadas con UNIVERSIA, además del consiguiente contraste y comprobación de la imagen que arroja la ortofoto actualizada, cada Universidad registrada se acompaña de datos espaciales para su 
geocodificación. Domicilio postal e identificación de coordenadas para cada uno de los diferentes espacios de concentración (campus con una o más Facultades e instancias universitarias como Bibliotecas, Aularios, Servicios Centrales y Periféricos, etc.) proporcionan los datos necesarios para georreferenciar cada caso. Tras varias iteraciones y filtros, casi todas las grandes ciudades españolas han acabado albergando al menos un centro universitario ${ }^{12}$, siendo el primer resultado de 48 universidades públicas que aglutinan en total 116 campus repartidos entre las diferentes áreas.

\section{d) Parques tecnológicos}

Anteriormente se ha mencionado la evolución seguida en los países desarrollados respecto a los espacios de la investigación. La génesis de esta especialización del espacio ocupado en dichas tareas se encuentra en Silicon Valley hacia 1947, germen de los subsiguientes centros de investigación que se han sucedido y extendido por todos los países.

Si bien en nuestro país estuvieron con frecuencia anclados institucionalmente a las Universidades, con los cambios acaecidos en los sistemas productivos se acentuó el estímulo al mundo científico, especialmente asociado a las ingenierías en todas sus variaciones. Conforme a los avatares sociopolíticos de nuestro país, hasta el último tercio del siglo $\mathrm{XX}$ no se podía reconocer claramente ese cambio pero a partir de entonces los parques y polígonos industriales compartieron localización (y en ocasiones daban el relevo) con nuevos centros de actividad donde se hacía patente el creciente protagonismo de la industria de bata blanca. En esas décadas y ya entrado el siglo XXI se podían reconocer en algunas de nuestras ciudades aquellas áreas o recintos claramente acotados, destinados al espacio de la investigación asociada al campo experimental, el ensayo de tecnologías y el desarrollo de procesos innovadores y las simulaciones virtuales para grandes cadenas de producción. De ese modo, los polígonos industriales fueron regenerándose, reubicándose y finalmente abriendo camino a nuevas centralidades generadas en torno a los centros y parques tecnológicos. De acuerdo a la investigación sobre estos espacios, es preciso diferenciar parque científico (de vocación universitaria u organismo público de investigación, casi al 100\%) del parque tecnológico, gestado a partir de la colaboración entre empresas, universidades y administración pública (Bueno, 2011). Al objeto de nuestro trabajo, una vez conocidas esas diferencias, en cada caso consideraremos que constituyen un mismo tipo de centralidad dada la convergencia en el aporte a la ciencia y la tecnología. En todo caso, estos nuevos elementos de la ciudad comparten tendencia con los anteriormente vistos, ya de más largo recorrido y cronología, y se tienden a localizaciones periféricas o incluso más allá de los bordes urbanos. La prospección visual sobre la ortofoto actualizada arroja en ocasiones datos sobre implantaciones en puntos más remotos de las coronas metropolitanas de lo que cabría pensar para otras actividades como el comercio o la sanidad. Estos matices ya comentados son oportunamente abordados en la siguiente fase del proyecto por parte de las autoras de este texto.

12 Solo dos áreas, Benidorm y Marbella, no albergan ningún campus ni sede de la Universidad Pública española. 


\section{a) Tratamiento de los datos}

La mayor parte de los parques tecnológicos en nuestro país están identificados como miembros de la Asociación de Parques Tecnológicos de España (A.P.T.E.), aun cuando en su orden interno diferencian aquellos que son socios de los otros miembros que no siéndolo al $100 \%$ están afiliados a dicha entidad.

En la búsqueda de los centros se detectó el solapamiento con las propias instituciones universitarias, que aparecían reflejadas como Campus «X», lo que en ocasiones se podría considerar un signo equívoco de la actividad desarrollan en estos parques. Ello nos condujo a afinar la caracterización de estos espacios hasta conocer claramente si se trataba de un recinto universitario, y de ser así, la variación funcional (investigación o docencia, o ambas) que acotara claramente el carácter del G.C.F.A.

Prácticamente todas las áreas analizadas cuentan al menos con un Parque Científico o Tecnológico. No es sorprendente que la mayor concentración se produzca en las grandes áreas metropolitanas, donde se encuentran no uno sino varios Parques Tecnológicos y con diferentes titularidades (públicas, privadas, mixtas). Si bien la selección de las universidades y los hospitales se aviene al criterio de titularidad pública, ese criterio no puede ser de aplicación en este caso, ya que se ha detectado la presencia de capital e instituciones públicas en ámbitos claramente identificados con firmas y lobbys de empresas privadas. En última instancia, como se ha dicho anteriormente, la investigación en materia de tecnología, nuevos materiales, nuevos sistemas, procesos, etc. converge -venga de donde venga- en la construcción de una realidad mejorada, donde la calidad ambiental es observada de un modo u otro (no en vano se potencia el concepto smart design and results en tantos campos de la investigación y la producción en la actualidad).

\section{b) Criterios de selección. Geolocalización}

De entre los 45 parques tecnológicos asociados ${ }^{13}$, se han computado todos aun cuando se identifica en determinados casos la superposición con complejos e instalaciones universitarias. Las cifras básicas tras la incorporación a nuestra base de datos son:

Tabla 4

CLASIFICACIÓN DE G.C.F.A. CON DESARROLLO DE PARQUES TECNOLÓGICOS EN LAS ÁREAS METROPOLITANAS ESPAÑOLAS

\begin{tabular}{cl}
\hline CANTIDAD & \multicolumn{1}{c}{ RANGO } \\
\hline TOTAL: 66 & $\mathbf{( 4 5 \text { SOCIOS Y 21 AFILIADOS) }}$ \\
\hline 19 & $<150.000 \mathrm{~m}^{2}$ \\
\hline 15 & $150.000-500.000 \mathrm{~m}^{2}$ \\
\hline 13 & $500.000-1.000 .000 \mathrm{~m}^{2}$ \\
\hline 6 & $1.000 .000-1.500 .000 \mathrm{~m}^{2}$ \\
\hline 6 & $>1.500 .000 \mathrm{~m}^{2}$ \\
\hline 7 & S/D (Se desconoce la superficie de la parcela) \\
\hline
\end{tabular}

Fuente A.P.T.E. Elaboración propia.

13 Estos centros han sido sacados de la web http://www.apte.org/es/parques-miembros.cfm, 


\section{e) Centros logísticos}

El actual sistema de producción, así como las pautas de consumo a diferentes escalas y sectores, hasta llegar al consumo doméstico final, han acabado por generar espacios lo suficientemente grandes y preparados para garantizar la distribución y almacenaje, todo ello ajustado a los tiempos requeridos en cada caso (Vahí, 2007). Los procesos de interacción derivados de la intensificación de flujos en el transporte de mercancías han sido cambiantes y progresivos, de ese modo se explica el cambio desde las áreas de almacenaje en los puertos, instalaciones ferroviarias o los seculares «mercas» que se podían ver en casi todas las capitales españolas, hacia el diseño y aplicación de criterios más complejos que conciben una estratégica organización como principal instrumento de la optimización en la actividad del transporte. Así se reconocen cada vez más centros logísticos localizados en relación a una distribución jerarquizada, en función de los puntos de carga/descarga y de los itinerarios que deben recorrer cada uno de los medios. El control que las mercancías superan a lo largo de los recorridos permite sentar la confianza en el traslado de productos de todo tipo a grandes distancias del lugar de procedencia. Todo ello ha suscitado la aparición de grandes centros logísticos ocupados en dar respuesta a la demanda con posibilidades de fragmentar, almacenar o/y transferir las cargas (Gutiérrez, García, 2010). Se pueden identificar en nuestro país cientos de centros logísticos de firmas comerciales, aunque nuestro cometido en una primera fase es abordar la identificación de los centros logísticos de carácter público o consorciado, a fin de determinar -igual que se ha hecho con los otros tipos de G.C.F.A.- su papel en el contexto urbano en que se insertan.

\section{a) Tratamiento de los datos}

La búsqueda de este tipo de centros se ha realizado sobre varias fuentes. Comenzando por la consulta a directorios del propio sector, se tomó como punto de referencia uno de ellos (www.centrosdetransporte.com) y a partir de ahí se han contrastado los datos con las webs sectoriales de los gobiernos autonómicos y también del Ministerio de Fomento. La suma de listados obtenidos se ha sometido a varios filtros a fin de evitar ciertas duplicidades en los registros y para depurar los centros no integrados en 46 las áreas metropolitanas.

\section{b) Criterios de selección. Geolocalización}

Los elementos registrados han sido seleccionados en virtud de la titularidad pública o participada de capital público y privado, de tal modo que generan una dinámica permanente y regular propia del suministro ha diversificado, en correspondencia con diferentes sectores, servicios y empresas. Por último, antes de geocodificar la información se procedió a la comprobación visual en la ortofoto actualizada ya aludida, a partir de la cual se podía constatar la fidelidad y veracidad de los datos recogidos. Los resultados, a primera vista, no resultan sorprendentes dada la correspondencia aproximada con el tamaño de la población y el volumen de los servicios y actividades. La mayor concentración de la oferta en Madrid (10) y Barcelona (5) y Zaragoza (5), y la progresiva disminución de centros en el resto de áreas ratifican lo dicho. Pero más allá de la distribución general sobre el mapa metropolitano del país, será importante determinar el alcance que estos centros de actividad tienen no sólo de cara al inte- 
rior de las áreas a las que sirven, sino en relación al exterior de las mismas. Las condiciones propias de la naturaleza de los centros (portuarios, ferroviarios,... intermodales) marcan la pauta para la localización definitiva del centro pero al mismo tiempo pueden llegar a dar juego para futuras proyecciones con el trazado de nuevos corredores industriales y empresariales.

Por último añadimos que la consiguiente identificación y/o vecindad de los centros logísticos con buena parte de las infraestructuras portuarias, aeroportuarias y ferroviarias simplifican la exploración inicial y las tareas subsiguientes de vinculación con las infraestructuras del viario.

\section{PRIMEROS RESULTADOS}

La mayoría de los G.C.F.A. registrados a fecha de 2013 eran inexistentes hace 30 años, sin embargo con su presencia han dado un vuelco a la dinámica de las actividades que en ellos se concentran, así como a la naturaleza y frecuencia de los desplazamientos urbanos. Incluso las tendencias de carácter morfoestructural que va trazando la ciudad metropolitana en su desarrollo y crecimiento se ven en mayor o menor medida influenciadas por la presencia de los grandes equipamientos y centros de actividad analizados. Las peculiaridades y requerimientos locacionales nos llevan a resultados interesantes que no conviene desdeñar. Veamos de manera separada cada uno de ellos en virtud del tratamiento sobre la localización y la identificación de los elementos registrados.

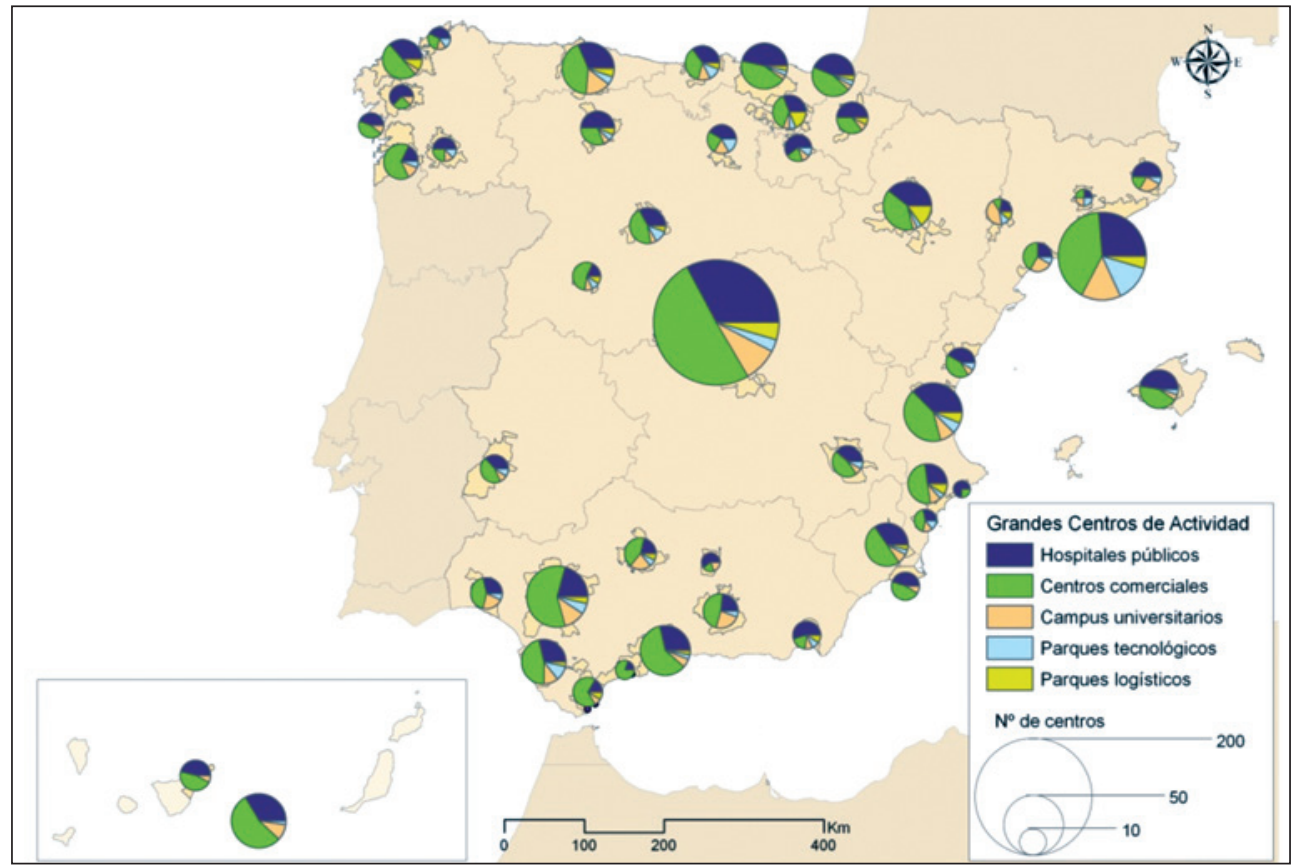

Fuente: Elaboración propia a partir de mapa base de España del IGN (fondo cartográfico). 
Entre las posibles aplicaciones de la explotación de la base de datos geográfica está la posibilidad de hacerlo mediante representación gráfica (uso de SIG). Teniendo en cuenta que la capacidad de atracción de los G.C.F.A. supera los límites administrativos tradicionales, parece más acertado en lo que se refiere a la representación e interpretación de los resultados, que la salida cartográfica se realice en base a criterios que permitan superar la representación según superficies discretas. En este sentido, resulta interesante hacerlo con base en índices asociados a la densidad en la distribución de los centros de actividad, aunque no renunciamos al análisis a partir de la evolución seguida por los centros. De hecho, un análisis diacrónico nos ofrece la oportunidad de un recorrido en perspectiva hasta reconocer el aumento de G.C.F.A. de acuerdo a un modelo económico que no siempre subsume la caracterización del territorio que lo acoge. El presente texto se ilustra con una selección de los primeros resultados obtenidos, referidos en este caso a las áreas metropolitanas de Andalucía y el comportamiento de los equipamientos comerciales (Tabla 5).

Tabla 5

EVOLUCIÓN DE LOS CENTROS COMERCIALES EN ANDALUCIIA SEGÚN SU S.B.A. Y SU SITUACIÓN CON RESPECTO A LA TRAMA URBANA (PORCENTAJES SOBRE EL TOTAL DE CENTROS DEL PERIODO)

\begin{tabular}{l|lrrr}
\hline \multicolumn{2}{c}{ Variable } & \multicolumn{3}{c}{ Año de apertura } \\
\hline \multirow{3}{*}{ S.B.A. $\left(\mathrm{m}^{2}\right)$} & $5.000-20.000$ & $\mathbf{1 9 9 0}$ & $\mathbf{1 9 9 1 - 2 0 0 0}$ & $\mathbf{2 0 0 1 - 2 0 1 2}$ \\
\cline { 2 - 5 } & $20.000-40.000$ & $22 \%$ & $59 \%$ & $42 \%$ \\
\cline { 2 - 5 } & $>40.000$ & $4 \%$ & $22 \%$ & $38 \%$ \\
\hline \multirow{2}{*}{ Situación territorial } & Intraurbano & $65 \%$ & $68 \%$ & $19 \%$ \\
\cline { 2 - 5 } & Interurbano & $35 \%$ & $32 \%$ & $52 \%$ \\
\hline \multicolumn{2}{l}{ Total centros comerciales } & $\mathbf{2 3}$ & $\mathbf{3 7}$ & $\mathbf{5 2}$ \\
\hline
\end{tabular}

Fuente: Elaboración propia a partir de Directorio de A.E.C.C. y otros.

La localización y consideración de estos G.C.F.A. como nodos permite, entre otros, el análisis espacio-temporal o diacrónico de los mismos, y por ende explorar la vinculación que este tipo de centros han podido tener a lo largo del tiempo en relación a la trama urbana. Buen ejemplo de ello son los resultados que se reflejan en la tabla 5, donde puede apreciarse como, en el caso concreto de los centros comerciales de Andalucía, se distingue una tendencia cada vez mayor hacia la apertura de centros de medianas y grandes dimensiones (S.B.A.), y a su vez una situación creciente de desapego o pérdida de vinculación con el resto de la trama urbana, y en especial con el tejido residencial ${ }^{14}$. Tendencia que se hace especialmente palpable en la última década, donde además se produce la apertura de prácticamente los mismos centros (52) que los que ya hay establecidos hasta el año 2001(60).

14 La diferenciación entre suelos interurbanos e intraurbanos está implícita en el desarrollo del planeamiento urbanístico. El Decreto-Ley de Comercio Interior de Andalucía 1/2013 se hace eco de ello de cara a la óptima ordenación comercial. 
La presencia creciente de centros comerciales hacia la periferia urbana, aun siguiendo pautas y ritmos expansivos variables en el tiempo, viene justificada (desde el punto de vista del mercado) por la búsqueda de parcelas y solares del tamaño necesario y a mejor precio que en el casco urbano. Ha sido y aún lo es una tendencia generalizada en las áreas metropolitanas españolas y la tendencia de las macrocifras que se conocen al respecto (S.B.A./1000 habitantes, número de grandes establecimientos, fórmulas de implantación, etc) indican que estos se hacen eco del diseño planificador y el modelo territorial establecido, algo sobre lo que es preciso profundizar a fin de contener el crecimiento y garantizar el desarrollo equilibrado de los sistemas urbanos y metropolitanos (Vahí, 2010).

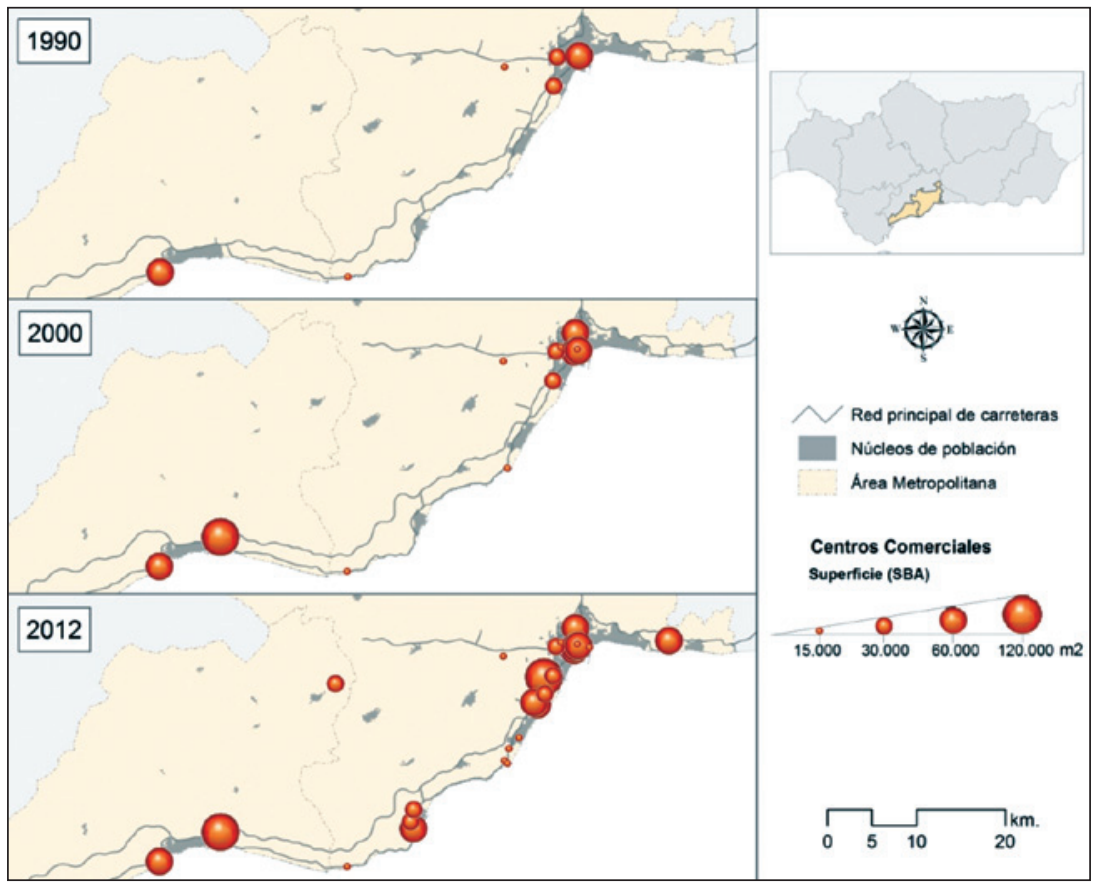

Fuente: elaboración propia.

Para el caso de la conurbación de las aglomeraciones de Málaga-Costa del Sol, esta tendencia se aprecia a simple vista, observándose el papel de las grandes redes de carretera como elemento guía para el establecimiento de estos centros (figura 4). La cartografía diacrónica expresa la previsible concentración de estos G.C.F.A. en los entornos urbanos más complejos (caso de las áreas metropolitanas) pero también el acompañamiento de las infraestructuras de las comunicaciones, que llega a convertirse ocasionalmente en el aliado idóneo para los procesos urbanos en expansión, tanto como de los intereses del mercado inmobiliario y terciarios. La cuestión no es baladí, sin embargo, ya que los resultados denotan que 
tanto los actores públicos como los privados obvian a menudo el carácter de rol organizador de dichos elementos infraestructurales sobre el medio ambiente y sobre el territorio en definitiva (Herce, 2010). De acuerdo con voces expertas en estos temas, las consecuencias no deseadas de un sistema que adolece de respuestas precisas (garantías de los flujos, accesos, conexiones,...) derivan de la errónea presunción de una lógica territorial (que debía prever estos G.C.F.A. en consonancia con la población demandante) y otra distinta de corte ambiental (que a menudo ha tenido un tratamiento correctivo o paliativo) para con estos grandes centros de servicios.

Si bien es cierto que los grandes establecimientos comerciales arrojan resultados muy claros en este sentido, el resto de las tipologías abordadas no escapan a la lógica del mercado de suelos en los entornos de las periferias urbanas y los intersticios dentro de las aglomeraciones y las áreas metropolitanas.

\section{CONCLUSIONES}

Las dinámicas espaciales observadas en las áreas metropolitanas españolas arrojan suficiente información para corroborar la relación existente entre usuarios y servicios, algunos de los cuales han desarrollado estrategias locacionales asociadas al tamaño de la oferta y, necesariamente, de los espacios que ocupan. Tal es el caso de los G.C.F.A., que en cualquiera de las tipologías abordadas (comercial, sanitaria, universitaria, tecnológica, logística) proporcionan junto a la satisfacción de la demanda para la que se crean, algunos conflictos o tensiones sobre el territorio sobre los que es necesario ahondar desde la perspectiva de la planificación y la coherencia. Dichos centros se han anclado al trazado de las grandes infraestructuras de las comunicaciones y los transportes, por lo que estos G.C.F.A. no se entienden si no son asociados básicamente a las redes de carreteras de primer nivel. Los grandes centros de actividad no solo ejercen su función como servicios especializados sino que acusan un rol inevitable en el esquema de la movilidad urbana metropolitana, algo que requiere mejoras que faciliten el buen funcionamiento de dichos centros y la operatividad de las redes. Para ello, la investigación en la que se inserta el presente artículo plantea bajo un enfoque sistémico la integración de los elementos intervinientes en el espacio metropolitano. La investigación sobre los G.C.F.A. arranca con una identificación exhaustiva y minuciosa de los elementos a partir del difícil equilibrio de criterios de diversificación y uniformidad que presentan. De ahí que el artículo haya planteado parte de los procedimientos contenidos en una metodología de la que no se conocen otros ensayos hasta el momento.

El análisis comparado de comportamientos de G.C.F.A. por tipologías, localización y áreas, permitirá detectar tendencias más o menos pautadas de zonificación para cada uno de los tipos, sobre la ciudad central, la $1^{\mathrm{a}}$ corona o $2^{\mathrm{a}}$ corona, o ambas, así como determinar el papel inductor de dichos centros sobre la aparición de elementos lineales como vías rápidas, corredores de comunicaciones, intercambiadores del transporte intermodal,... Así mismo, se probará a ensayar con cruces de bases de datos y unión de capas con información relativa al sistema de asentamientos (jerarquía de núcleos de población, urbanización dispersa...) que conduzcan, en la misma línea, a reconocer el diálogo de los G.C.F.A.con los elementos que les rodean, si son inductores o la consecuencia de una planificación y una demanda paulatina. La apreciación del acierto de la metodología empleada, donde 
las fuentes y los instrumentos utilizados han ido abriendo sucesivamente las puertas y los ensayos para probar continuamente con todos los recursos disponibles con garantías de fiabilidad. La dimensión del proyecto, la diversidad tipológica del objeto de estudio y la casuística en la que se sumen los grandes centros de actividad no impiden avanzar sobre el conocimiento de estos elementos, sus dinámicas y el papel que desempeñan sobre el conjunto de la realidad urbana y metropolitana. El carácter de centralidad que han trasladado a las periferias y los territorios metropolitanos, convierte a los G.C.F.A. en objeto de observación en tanto que adoptan un fuerte protagonismo junto a otros usos de suelo, a procesos de crecimiento urbano y, en definitiva, a la compleja movilidad cotidiana. La dotación de sistemas generales, entre los que las infraestructuras de las comunicaciones ocupan lugar preeminente se contienen igualmente en este esquema complejo de la realidad metropolitana que demanda una permanente atención y revisión/reconducción de las políticas urbanas sobre las experiencias adquiridas.

\section{BIBLIOGRAFÍA}

AECC (2010): Directorio de Centros Comerciales. Madrid. Asociación Española de Centros Comerciales.

ARROYO, M. (2001): «La contraurbanización: un debate metodológico y conceptual sobre la dinámica de las áreas metropolitanas», Scripta Nova. nº 97.

BORRUSO, G. (2003): «Network density and the Delimitation of Urban Areas», Transactions in GIS, 7 (2), 177-191.

BUENO, E. (2011): «Los Parques Científicos y Tecnológicos como espacios de encuentro multidisciplinar para la innovación», Encuentros multidisciplinares, vol. 13, n ${ }^{\circ} 37$, 2011 (Ejemplar dedicado a: Los parques científicos como espacios multidisciplinares), 26-35.

CARRERAS, C. (1995): «Los nuevos espacios del consumo en Barcelona», Revista de Geografía, $\mathrm{n}^{\mathrm{o}}$ 29, 2, (Ejemplar dedicado a: Terciarización y consumo en las ciudades de Barcelona y Lisboa), 69-78.

CASADO, J.M. y COOMBES, M. (2005): «The Delineation of $21^{\text {st }}$ Century Local Labour Market Areas (LLMAs)», $8^{\text {th }}$ NECTAR Conference, Universidad de Las Palmas, Las Palmas de Gran Canaria, http://www.nectarlaspalmas.com/ , 2/VI/2005-4/VI/2005.

COMISIÓN EUROPEA (1992): Libro Blanco sobre el curso futuro de la política común de transportes COM(92) (0494).

COMISIÓN EUROPEA (1995): La política común de transportes - Programa de acción 1995-2000» COM(95) (0302).

COMISIÓN EUROPEA (1996): Libro Blanco - Estrategia para la revitalización de los ferrocarriles comunitarios $\mathrm{COM}(96)$ (421).

COMISIÓN EUROPEA (2007): Libro Verde - Hacia una nueva cultura de la movilidad urbana, $\operatorname{COM}(2007)(551)$.

COMISIÓN EUROPEA (2008): Libro Verde sobre la cohesión territorial - Convertir la diversidad territorial en factor de éxito $\operatorname{COM}(2008)$ (616).

ESCUDERO, L.A. (2008): Centros Comerciales, espacios posmodernos de ocio y consumo: un estudio geográfico. Cuenca. Universidad de Castilla-La Mancha, 2008. 
EUROPEAN ENVIRONMENT AGENCY (2004): Corine Land Cover Spain, CNIG.

FARINÓS, J. (2008): «Gobernanza territorial para el desarrollo sostenible: Estado de la cuestión y agenda», Boletín de la Asociación de Geógrafos Españoles, n 46, 11-32.

FERIA, J.M. (2004): «Problemas de definición de las áreas metropolitanas en España», Boletín de la Asociación de Geógrafos Españoles, nº 38, 85-99.

FERIA, J.M. (2008): «Un ensayo metodológico de definición de las áreas metropolitanas en España a partir de la variable residencia-trabajo», Investigaciones Geográficas, $\mathrm{n}^{\circ} 46$, 2008, 49-68.

FERIA, J.M. y ALBERTOS, J.M. (Coords.) (2010): La ciudad metropolitana en España: procesos urbanos en los inicios del siglo XX. Civitas-Thomson Reuters.

FERIA, J.M. y CARAVACA, I. (1994): «Universidad y ciudad: necesidad de un nuevo modelo espacial para la Universidad de Sevilla», Anales de Geografía de la Universidad Complutense, $\mathrm{n}^{\circ} 14,1994,105-118$.

FERIA, J.M. y SUSINO, J. (1996): Movilidad por razón de trabajo en Andalucía. Sevilla. Instituto de Estadística de Andalucía.

FERNÁNDEZ, J.C., GARCÍA-LEÓN, F.J., MOYANO, M.D., PUERTO, E.M., RODRÍGUEZ, V., RODRÍGUEZ, E. y RUIZ, M. (2011): «SIG Mercator de la Consejería de Salud de la Junta de Andalucía», Mapping, n 148, 2011, 62-65.

GUTIÉRREZ, J. y GARCÍA, J.C. (2010): «Procesos de descentralización de la actividad en el Área Metropolitana de Madrid», en La ciudad metropolitana en España: procesos urbanos en los inicios del siglo XXI (Feria, J.M., Albertos, J.M., Coords.), Civitas-Thomson Reuters, 241-273.

HERCE, M.: «Infraestructuras de transporte e impacto territorial: Métodos de planificación e implicaciones ambientales», en Herce Vallejo, M. (ed.): Infraestructuras y medio ambiente I. Urbanismo, territorio y redes de servicios. UOC, Barcelona, 2010.

MECD, Ministerio de Educación, Cultura y Deporte (Gobierno de España): Universidades. Datos generales http:/www.mecd.gob.es/educacion-mecd/areas-educacion/universidades/estadisticas-informes/datos-generales.html (consultado 2012)

MINISTERIO DE FOMENTO (2007): Atlas estadístico de las áreas urbanas españolas. Madrid, Centro de Publicaciones del Ministerio de Fomento.

MSSSI, Ministerio de Sanidad, Servicios Sociales e Igualdad (Gobierno de España):

- Instituto de Información Sanitaria (2012): «Ordenación Sanitaria del Territorio en las comunidades autónomas. Sistema de Información de Atención Primaria (SIAP). Año 2012». Madrid.

- Catálogo de Centros de Atención Primaria de SNS, Catálogo Nacional de Hospitales (2012). http://www.mspsi.es/estadEstudios/estadisticas/estadisticas/estMinisterio/siap.html

- Portal estadístico de hospitales: Estadística de establecimientos sanitarios con régimen de internado (ESCRI). Glosario de términos y definiciones (consultado 2012). http://www.msc.es/ciudadanos/prestaciones/centrosServiciosSNS/hospitales/home.htm

MÉNDEZ, R. y RODRÍGUEZ, J.M. (2007): «Transformaciones productivas y nuevas formas urbanas: difusión de las actividades económicas en la región metropolitana funcional de Madrid», Anales de Geografía de la Universidad Complutense, Vol. 27, n 2, 2007, 105-134. 
MORENO, A. (1991): «Modelización cartográfica de densidades mediante estimadores Kernel». Treballs de la Societat Catalana de Geografía, 6, 30, 155-170.

OLLERO, M., (2004): «Unidades de estancia corta y adecuación del ingreso hospitalario», Medicina Clínica, 123(16), 621-623.

POZO, E., RUIZ, E. y LÁZARO, M.L. (2000): «Nuevas formas de comercio y consumo en Madrid: las grandes superficies», Estudios Geográficos, Vol. 61, n 238, 125-144.

REQUES, P. (Dir.) (2006): Atlas digital de la España Universitaria. Bases para la planificación estratégica de la enseñanza superior. Universidad de Cantabria. Banco de Santander.

RODRÍGUEZ, V. (2010): «Medición de la accesibilidad geográfica de la población a la red de hospitales de alta resolución de Andalucía mediante Sistemas de Información Geográfica», Ojeda, J., Vallejo, I., Pita, M.F. (Coords.): La información geográfica al servicio de los ciudadanos: de lo global a lo local. Universidad de Sevilla, 549-564.

ROMERO, J. y CARRERAS, C. (2000): «Cambios en las formas del comercio y el consumo en Barcelona», Estudios geográficos, Vol. 61, N 238, 2000, 103-124.

SALOM, J. y ALBERTOS, J.M. (2010): «Metodología para la delimitación del espacio metropolitano a partir de la densidad del viario» en Benito del Pozo, P.(coord.): Las nuevas áreas empresariales: promoción y recualificación del suelo industrial, logística y gobernanza: comunicaciones. Madrid. Asociación de Geógrafos Españoles, Grupo de Geografía Económica, 1- 20.

SANZ, L. y CRUZ, L. (2001): «Autonomía y adaptación organizativa: los centros de investigación ante los cambios del entorno» Reis, Revista Española de Investigaciones Sociológicas, $\mathrm{n}^{\circ}$ 95, 37-67.

TORNOS, J. (Coord.) (2000): Grandes establecimientos comerciales: su ordenación e implantación, Universidad Autónoma de Barcelona, Servei de Publicacions.

UNIVERSIA, Portal de las Universidades Españolas (Mecenazgo de Banco de Santander), http://estudios.universia.net/espana/institucion (consultado 2012).

VAHÍ, A. (2007): La perspectiva territorial y urbana de los grandes equipamientos comerciales en Andalucía. Sevilla, Universidad de Sevilla-Consejería de Obras Públicas y Transportes, Junta de Andalucía.

VAHÍ, A. y FERIA, J.M. (2007): «Estructuras urbanas y grandes formatos comerciales: el ejemplo de las áreas metropolitanas andaluzas», Ería: Revista cuatrimestral de geografía, $\mathrm{n}^{\mathrm{o}} 72,2007,35-54$.

VAHÍ, A. (2010): «El territorio y la orientación comercial. La salvaguarda del patrimonio territorial en las Comunidades Autónomas de España», Anales de Geografía, Universidad Complutense, Vol. 30, $\mathrm{n}^{\circ}$. 1, 139-161.

VILLAREJO, H. y ÁLVAREZ, S. (2004): «Causas y consecuencias de la intervención pública en los grandes escenarios de consumo», Estudios sobre consumo, n ${ }^{\circ}$ 69, 2004, 9-28.

VINUESA, J. (2012): «Procesos y estructuras geodemográficas en la metropolitanización. Propuesta de debate sobre algunas cuestiones básicas», en Reques, P. y De Cos, O.: La población en clave territorial. Procesos, estructuras y perspectivas de análisis (XIII CONGRESO DE LA POBLACIÓN ESPAÑOLA, Santander, 2012).

ZAMBRANA, J.L., JIMÉNEZ-OJEDA, B., MARÍN, M. y ALMAZÁN S. (2002): «La consulta única o de alta resolución como una alternativa de eficiencia a las consultas externas hospitalarias tradicionales», Med Clin; Vol. 119, 302-5. 
